\title{
Editorial: Mechanisms of Fluorescent Proteins
}

\author{
Chong Fang ${ }^{1 *}$, Mikhail Drobizhev ${ }^{2 *}$, Ho Leung $\mathrm{Ng}^{3 *}$ and Periklis Pantazis ${ }^{4 *}$ \\ ${ }^{1}$ Department of Chemistry, Oregon State University, Corvallis, OR, United States, ${ }^{2}$ Department of Cell Biology and Neuroscience, \\ Montana State University, Bozeman, MT, United States, ${ }^{3}$ Department of Biochemistry and Molecular Biophysics, Kansas State \\ University, Manhattan, KS, United States, ${ }^{4}$ Department of Bioengineering, Imperial College London, London, United Kingdom
}

Keywords: genetically encoded markers, photophysics and photochemistry, chromophore structure and dynamics, bioimaging and engineering, molecular machineries, noncanonical amino acid, ultrafast spectroscopy

Editorial on the Research Topic

\section{Mechanisms of Fluorescent Proteins}

This collection of papers for the Research Topic "Mechanisms of Fluorescent Proteins" (FPs) samples a broad range of research on physical mechanisms, applications, and molecular engineering strategies. The papers demonstrate a combination of experimental and computational approaches and are of broad interest to researchers working on FPs, microscopy, and spectroscopy.

In bioimaging with FPs, increasing the penetration depth and decreasing unwanted scattering are

\section{OPEN ACCESS}

Edited and reviewed by: Neil Marsh, University of Michigan, United States

*Correspondence: Chong Fang chong.fang@oregonstate.edu Mikhail Drobizhev mikhail.drobijev@montana.edu Ho Leung Ng hng@ksu.edu Periklis Pantazis p.pantazis@imperial.ac.uk

Specialty section: This article was submitted to Protein Chemistry and Enzymology, a section of the journal

Frontiers in Molecular Biosciences

Received: 28 April 2021 Accepted: 10 May 2021 Published: 26 May 2021

Citation:

Fang C, Drobizhev M, Ng HL and Pantazis P (2021) Editorial: Mechanisms of Fluorescent Proteins.

Front. Mol. Biosci. 8:701523. doi: 10.3389/fmolb.2021.701523 desirable, which have motivated efforts for engineered FPs with redder emission and higher brightness (Subach and Verkhusha, 2012; Dedecker et al., 2013). In a combined experimental and computational work (Gorbachev et al., 2020), the green/red photoconversion of EGFP with reducing agents was investigated and a novel green-emitting state only present under low-oxygen conditions was identified. Following photoconversion, the distinct orange and red-emitting forms (565 and $600 \mathrm{~nm}$ emission maxima) differ from the reported red-emitting form (607 $\mathrm{nm}$ emission) via oxidative reaction. This work showcases a complex interplay between the chromophore and protein environment, generating a neutral quinoid-like green-emitting chromophore $(525 \mathrm{~nm}$ emission) as an intermediate. This step leads to a zwitterionic form of the photoexcited chromophore via charge transfer that bifurcates into the orange and red-emitting forms. Such a general oxidative mechanism enriches the FP application toolset (Bourgeois and Adam, 2012; Jung, 2012; Krueger et al., 2020; Nasu et al., 2021) with additional tunable "knobs" of oxygen levels and redox-active compounds to control photoconversion and achieve redder emission.

To brighten the generally dim red and far-red FPs, a systematic study of nonradiative relaxation in red FPs (RFPs) (Drobizhev et al., 2021) reveals a dominant role of the twisted intramolecular charge transfer mechanism over the energy gap law. This work substantiates local electrical field control of fluorescence quantum yield (FQY) of RFPs. Aided by one- and two-photon absorption spectroscopy and quantum calculations of seven different RFPs with the same chromophore structure, a spectroscopic method of evaluating local electric fields (amplitude and direction in $E_{x}$ and $E_{y}$ ) at the protein chromophore enables separation of contributing factors to the nonradiative relaxation rate. A small range of positive or negative values for $E_{x}$ and $E_{y}(-10$ to $+10 \mathrm{MV} / \mathrm{cm})$ was revealed to facilitate both a red-shifted absorption and a high FQY, providing rational design principles for sitespecific mutagenesis using RFP scaffold like DsRed.

On a fundamental level, the fluorescence mechanism via excited-state proton transfer (Chattoraj et al., 1996; Fang et al., 2009; Tonge and Meech, 2009; Fang and Tang, 2020) is elucidated further by a computational study (Coppola et al., 2020) on the complex hydrogen (H)-bond equilibrium dynamics for neutral, intermediate, and anionic chromophore forms inside GFP. An accurate 
hybrid QM/MM simulation of the entire protein was performed to enable the intricate correlation between chromophore site-specific single $\mathrm{H}$-bond interactions and the chromophore cavity volume, and noncovalent interactions with distant residues on opposite sides of the pocket. This work showcases the power of $a b$ initio molecular dynamics simulations in hybrid form with density functional theory (DFT) to bridge local and larger-scale effects in FPs.

Most of FPs contain a $\pi$-conjugated chromophore, p-hydroxybenzylidene-imidazolinone (HBDI). Typical examples are GFP from Aequorea victoria, and DsRed from Discosoma sp. wherein the HBDI chromophore is amended by an acylimine tail that lengthens $\pi$-conjugation and red-shifts the absorption. Targeted engineering for brighter variants was typically driven by linear, onephoton absorption and fluorescence. With the advent of two-photon laser microscopy, the need for bright and photostable FPs has increased. Yet two-photon absorption obeys different quantummechanical rules compared with one-photon absorption. Consequently, the brightest FPs with canonical chromophores for one-photon imaging are not necessarily optimized for two-photon excitation. The two-photon cross-sections of ten non-canonical chromophores (nCCs), inserting substituted tyrosines into the DsRed scaffold, were calculated using QM/MM schemes with polarizable embedding and external effective field correction (Rossano-Tapia et al., 2020). Although none of the model proteins shows a two-photon cross-section larger than DsRed (List et al., 2016), the work helps to understand structurefunction relationships and design better two-photon-absorbing FPs.

Three other FPs with nCCs containing $\mathrm{Cl}-, \mathrm{Br}-$, and nitrosubstituted tyrosine were prepared from sfGFP scaffold and studied using a combination of femtosecond transient absorption and stimulated Raman spectroscopy (FSRS) (Oscar et al., 2020). The FSRS measurements were supported by DFT calculations of vibration normal modes for accurate assignments. The high spectral and temporal resolution obtained by FSRS and transient absorption allowed delineation of the chromophore protonation state and isomeric structure. Longer vibrational relaxation times in the excited state of Cl-sfGFP (4 ps) and Br-sfGFP (11 ps), compared with the parent sfGFP (1.2 ps), were correlated with the increased FQY. Moreover, FPs with halogenated chromophores exhibit advantageous redshifts in their absorption and emission spectra, rendering them great candidates for bioimaging applications (Pantazis and Supatto, 2014).

Among the most exciting applications of FPs is their use in single-molecule and super-resolution fluorescence microscopy.

\section{REFERENCES}

Bourgeois, D., and Adam, V. (2012). Reversible Photoswitching in Fluorescent Proteins: A Mechanistic View. IUBMB Life 64 (6), 482-491. doi:10.1002/iub.1023 Chalfie, M., Tu, Y., Euskirchen, G., Ward, W., and Prasher, D. (1994). Green Fluorescent Protein as a Marker for Gene Expression. Science 263 (5148), 802-805. doi:10.1126/science.8303295

Chattoraj, M., King, B. A., Bublitz, G. U., and Boxer, S. G. (1996). Ultra-Fast Excited State Dynamics in Green Fluorescent Protein: Multiple States and Proton Transfer. Proc. Natl. Acad. Sci. USA 93 (16), 8362-8367. doi:10.1073/pnas.93. 16.8362
Such applications necessitate FPs with superior properties such as high FQY, fraction of time remaining fluorescent, outstanding photostability, and structural stability (Bourgeois and Adam, 2012; Nienhaus and Nienhaus, 2016; Woodhouse et al., 2020). In this issue, a bilirubin-activated photoswitching protein called eUnaG was developed with the highest bulk fluorescence to date, comparable to organic dyes (Ko et al., 2021). The superior performance of eUnaG is primarily due to its increased stability, leading to reduced aggregation and fewer labeling artifacts. eUnaG promises to support state-of-the-art performance for high-resolution microscopy.

From this exciting line of inquiries decoding fluorescence mechanisms of FPs, particularly targeting redder and brighter emissions, we foresee the interdisciplinary spectroscopy, microscopy, theoretical, and computational communities to continue joining forces to paint a comprehensive portrait of FPs and implement these molecular machines in everexpanding applications. Nature has evolved FPs for millions of years, while GFP has revolutionized molecular and cellular biology just for several decades (Shimomura et al., 1962; Chalfie et al., 1994; Tsien, 1998). Much remains to be learned and developed, and we hope this special Research Topic in Front. Mol. Biosci. (https://www.frontiersin.org/research-topics/ 10542/mechanisms-of-fluorescent-proteins\#articles) has captured the essence of this field and will inspire future innovations and breakthroughs in both the understanding and applications of FPs.

\section{AUTHOR CONTRIBUTIONS}

All authors listed have made a substantial, direct, and intellectual contribution to the work and approved it for publication.

\section{FUNDING}

CF acknowledges the US National Science Foundation (NSF) grants MCB-1817949 and CHE-2003550. MD acknowledges the NIH/NINDS BRAIN grants U01 NS094246 and U24 NS109107. HN was funded by the US NSF CAREER Award MCB-1833181. PP is a Royal Society Wolfson Research Merit Award holder and acknowledges support from the BBSRC (BB/T017929/1 and BB/ T011947/1).

Coppola, F., Perrella, F., Petrone, A., Donati, G., and Rega, N. (2020). A Not Obvious Correlation between the Structure of Green Fluorescent Protein Chromophore Pocket and Hydrogen Bond Dynamics: A Choreography from Ab Initio Molecular Dynamics. Front. Mol. Biosci. 7, 569990. doi:10.3389/fmolb.2020.569990

Dedecker, P., De Schryver, F. C., and Hofkens, J. (2013). Fluorescent Proteins: Shine on, You Crazy Diamond. J. Am. Chem. Soc. 135 (7), 2387-2402. doi:10.1021/ja309768d

Drobizhev, M., Molina, R. S., Callis, P. R., Scott, J. N., Lambert, G. G., Salih, A., et al. (2021). Local Electric Field Controls Fluorescence Quantum Yield of Red and Far-Red Fluorescent Proteins. Front. Mol. Biosci. 8, 633217. doi:10.3389/fmolb.2021.633217

Fang, C., and Tang, L. (2020). Mapping Structural Dynamics of Proteins with Femtosecond Stimulated Raman Spectroscopy. Annu. Rev. Phys. Chem. 71 (1), 239-265. doi:10.1146/annurev-physchem-071119-040154 
Fang, C., Frontiera, R. R., Tran, R., and Mathies, R. A. (2009). Mapping GFP Structure Evolution during Proton Transfer with Femtosecond Raman Spectroscopy. Nature 462 (7270), 200-204. doi:10.1038/nature08527

Gorbachev, D. A., Petrusevich, E. F., Kabylda, A. M., Maksimov, E. G., Lukyanov, K. A., Bogdanov, A. M., et al. (2020). A General Mechanism of Green-to-Red Photoconversions of GFP. Front. Mol. Biosci. 7, 176. doi:10.3389/fmolb.2020.00176

G. Jung (2012). in Fluorescent Proteins II: Application of Fluorescent Protein Technology (Berlin Heidelberg: Springer-Verlag)

Ko, S., Kwon, J., and Shim, S.-H. (2021). Enhanced UnaG with Minimal Labeling Artifact for Single-Molecule Localization Microscopy. Front. Mol. Biosci. 8, 647590. doi:10.3389/fmolb.2021.647590

Krueger, T. D., Tang, L., Zhu, L., Breen, I. L., Wachter, R. M., and Fang, C. (2020). Dual Illumination Enhances Transformation of an Engineered Green-to-Red Photoconvertible Fluorescent Protein. Angew. Chem. Int. Ed. 59 (4), 1644-1652. doi:10.1002/anie.201911379

List, N. H., Jensen, H. J. A., and Kongsted, J. (2016). Local Electric Fields and Molecular Properties in Heterogeneous Environments through Polarizable Embedding. Phys. Chem. Chem. Phys. 18 (15), 10070-10080. doi:10.1039/ $\mathrm{C} 6 \mathrm{CP} 00669 \mathrm{H}$

Nasu, Y., Shen, Y., Kramer, L., and Campbell, R. E. (2021). Structure- and Mechanism-Guided Design of Single Fluorescent Protein-Based Biosensors. Nat. Chem. Biol. 17, 509-518. doi:10.1038/s41589-020-00718-x

Nienhaus, K., and Nienhaus, G. U. (2016). Photoswitchable Fluorescent Proteins: Do Not Always Look on the Bright Side. ACS Nano 10 (10), 9104-9108. doi:10. 1021/acsnano.6b06298

Oscar, B. G., Zhu, L., Wolfendeen, H., Rozanov, N. D., Chang, A., Stout, K. T., et al. (2020). Dissecting Optical Response and Molecular Structure of Fluorescent Proteins with Non-canonical Chromophores. Front. Mol. Biosci. 7, 131. doi:10. 3389/fmolb.2020.00131

Pantazis, P., and Supatto, W. (2014). Advances in Whole-Embryo Imaging: A Quantitative Transition Is Underway. Nat. Rev. Mol. Cel Biol. 15 (5), 327-339. doi:10.1038/nrm3786
Rossano-Tapia, M., Olsen, J. M. H., and Brown, A. (2020). Two-Photon Absorption Cross-Sections in Fluorescent Proteins Containing Noncanonical Chromophores Using Polarizable QM/MM. Front. Mol. Biosci. 7, 111. doi:10.3389/fmolb.2020.00111

Shimomura, O., Johnson, F. H., and Saiga, Y. (1962). Extraction, Purification and Properties of Aequorin, a Bioluminescent Protein from the Luminous Hydromedusan, Aequorea. J. Cell. Comp. Physiol. 59 (3), 223-239. doi:10. 1002/jcp.1030590302

Subach, F. V., and Verkhusha, V. V. (2012). Chromophore Transformations in Red Fluorescent Proteins. Chem. Rev. 112 (7), 4308-4327. doi:10.1021/cr2001965

Tonge, P. J., and Meech, S. R. (2009). Excited State Dynamics in the Green Fluorescent Protein. J. Photochem. Photobiol. A: Chem. 205 (1), 1-11. doi:10. 1016/j.jphotochem.2009.03.021

Tsien, R. Y. (1998). The Green Fluorescent Protein. Annu. Rev. Biochem. 67 (1), 509-544. doi:10.1146/annurev.biochem.67.1.509

Woodhouse, J., Nass Kovacs, G., Coquelle, N., Uriarte, L. M., Adam, V., Barends, T. R. M., et al. (2020). Photoswitching Mechanism of a Fluorescent Protein Revealed by Time-Resolved Crystallography and Transient Absorption Spectroscopy. Nat. Commun. 11 (1), 741. doi:10. 1038/s41467-020-14537-0

Conflict of Interest: The authors declare that the research was conducted in the absence of any commercial or financial relationships that could be construed as a potential conflict of interest.

Copyright (C) 2021 Fang, Drobizhev, Ng and Pantazis. This is an open-access article distributed under the terms of the Creative Commons Attribution License (CC BY). The use, distribution or reproduction in other forums is permitted, provided the original author(s) and the copyright owner(s) are credited and that the original publication in this journal is cited, in accordance with accepted academic practice. No use, distribution or reproduction is permitted which does not comply with these terms. 